\title{
A PERSISTENT SCATTERER INTERFEROMETRY PROCEDURE TO MONITOR URBAN SUBSIDENCE
}

\author{
M. Crosetto ${ }^{1, *}$, O. Monserrat ${ }^{1}$, A. Barra ${ }^{1}$, M. Cuevas-González ${ }^{1}$, V. Krishnakumar ${ }^{1}$, M. Mróz ${ }^{2}$, B. Crippa ${ }^{3}$ \\ ${ }^{1}$ Centre Tecnològic de Telecomunicacions de Catalunya (CTTC/CERCA), Division of Geomatics, 08860 Castelldefels (Barcelona), \\ Spain ITU (mcrosetto, omonserrat, mcuevas, abarra, vkrishnakumar)@cttc.cat \\ ${ }^{2}$ Institute of Geodesy, University of Warmia and Mazury in Olsztyn, 10719 Olsztyn, Poland - marek.mroz@uwm.edu.pl \\ ${ }^{3}$ University of Milan, Department of Earth Sciences, 20129 Milan, Italy - bruno.crippa@unimi.it
}

Commission III, WG III/3

KEY WORDS: Radar, interferometry, atmospheric component, estimation, monitoring

\begin{abstract}
:
This paper describes a Persistent Scatterer Interferometry procedure for deformation monitoring. Its more original part concerns an approach to estimate the atmospheric phase component. The procedure can be used to monitor deformation areas that are relatively small and are surrounded by stable areas. The proposed procedure is described step by step. The procedure can be applied using SAR data coming from different sensors. However, in this work we discuss results obtained using Sentinel-1 data. A case study is described, where the deformation is caused by water pumping associated with construction works. In this case study, a stack of 78 Sentinel-1 images were analysed. The main part of the paper concerns the analysis of the atmospheric component. A comprehensive characterization of this component is first described, considering the original non-filtered phases. This is followed by the characterization of the residual filtered phases. This analysis highlights the goodness of the proposed procedure. This is further confirmed by the analysis of two deformation time series. The procedure can work with any type of deformation phenomena, provided that its spatial extension is sufficiently small.
\end{abstract}

\section{INTRODUCTION}

In this paper we describe a Persistent Scatterer Interferometry (PSI) procedure, which can be used to monitor deformation areas that are relatively small and are surrounded by stable areas. For a review of PSI see Crosetto et al. (2016). The procedure can be applied using SAR data coming from sensors working in different bands; however, in this work we discuss results that were based on Sentinel-1A and Sentinel-1B SAR data.

Sentinel-1 is a two-satellite mission of the Copernicus Programme of the European Union (www.copernicus.eu). The sensors on board the Sentinel-1 satellites acquire C-band Synthetic Aperture Radar (SAR) data. Compared to previous missions of the European Space Agency (ERS-1/2 and Envisat), Sentinel-1 offers improved performances in term of revisiting time, spatial coverage and quality of the SAR imagery. The Sentinel-1 satellites make mainly use of the Interferometric Wide Swath (IWS) acquisition mode, which provides a $250-\mathrm{km}$ image swath. They offer a repeat cycle of 6 days (considering both Sentinel-1A and 1B). The Sentinel-1 mission has been especially designed for massive wide-area monitoring. An important advantage of the Sentinel-1 mission is that the data are freely available for both scientific and commercial applications.

The first Sentinel-1A data were acquired in 2014. Since then several studies have been devoted to urban deformation monitoring. We mention in the following some examples that concern the deformation monitoring of entire metropolitan areas. They include Mexico City (Sowter et al., 2016), Madrid (Bakon et al., 2016), Shanghai (Yu et al., 2017), San Francisco (Shirzaei et al., 2017), Beijing (Du et al., 2018), Ravenna (Fiaschi et al., 2018) and Istanbul (Aslan et al., 2018).
This is the content of this paper. Section 2 describes the main steps of the proposed PSI procedure. The procedure has been applied to study the deformation associated with the construction works of a tunnel. Section 3 describes the case study and discusses the estimation of the atmospheric component of the PSI observations. Section 4 briefly describes the main results of the deformation monitoring in the analysed test case. Section 5 includes the conclusions of the work.

\section{PROPOSED PSI PROCEDURE}

The original part of the proposed procedure concerns the estimation of the atmospheric phase component. The PSI approaches usually make use of sets of spatial and temporal filters to estimate the atmospheric phase component, e.g. see Ferretti et al. (2000; 2001) and Berardino et al. (2002). A critical issue is the separation of the two main low-pass spatial contributions: the low-pass deformation and the atmospheric component. The assumption usually employed is that the former one is temporally correlated, while the latter component is not correlated in time. The first limitation of this approach is the difficulty to properly separate subtle deformation from the atmospheric contribution, with the risk to underestimate the former one. In addition, the deformation can sometimes be not so smooth in time. For instance, in some cases, the water pumping can be intermittent, implying a series of ups and downs of the ground.

The proposed procedure overcomes the above limitation. It does not make use of spatio-temporal filters and, more importantly, does not make any assumption regarding the temporal correlation

\footnotetext{
* Corresponding author
} 
of the deformation. It has two advantages: we can correctly estimate sudden deformation, without the disadvantage of filtering out the high-frequency temporal components of the deformation; in addition, we can avoid the subjective decisions often associated with spatio-temporal filtering.

In the proposed procedure the atmospheric component is estimated using stable areas that surround the deformation area at hand. Identifying stable areas requires external information; however, this assumption can be validated during the data analysis. The stable areas are used to estimate the atmospheric component, which is then predicted and removed from the PSI observations over the area of interest. Several approaches can be used for this purpose, e.g. kriging, least squares collocation, polynomials, etc.

In the following we describe step by step the proposed procedure. This sequence has been used to perform the case study. More details on the general PSI procedure adopted by the authors can be found in Crosetto et al. (2011) and Devanthéry et al. (2014).

Acquisition of a set of $\mathrm{N}$ interferometric Sentinel-1 SAR images that cover the area of interest. Usually a minimum of 20-25 images are used.

Precise co-registration of the entire burst stack that covers the area of interest. This is basically based on the information provided by the precise orbits associated with the images.

Generation of two redundant networks of interferograms. In the case study at hand, a full-resolution (pixel footprint: 4 by $14 \mathrm{~m})$ and 10 in range by 2 in azimuth (10x2) multi-look (pixel footprint: 40 by $28 \mathrm{~m}$ ) have been generated.

Pixel selection (the selected pixels are called candidate PSs - Persistent Scatterers) using the Amplitude Dispersion index proposed by Ferretti et al. (2000; 2001).

A 2+1D phase unwrapping is run on the redundant set of $10 \times 2$ multi-look interferograms. The algorithm is described in Devanthéry et al. (2014).

Identification of stable areas in the surroundings of the area of interest areas.

Estimation of the atmospheric phase component over the stable areas. In the case study described in this paper, this step has been performed assuming a linear phase model.

Prediction of the atmospheric phase component over the deformation area at hand.

Removal of the estimated atmospheric component. This operation is performed on the original single-look interferograms.

Estimation of the linear deformation velocity and residual topographic error using the method of the periodogram, see for details Biescas et al. (2007). This operation is performed using the set of atmospheric-free singlelook interferograms.

Removal of the residual topographic component from the atmospheric-free single-look interferograms.

A second 2+1D phase unwrapping is performed on the set of single-look interferograms (after removing the residual topographic component and the atmospheric component).
Generation of the final results and their geocoding, obtaining the deformation velocity and the deformation time series.

\section{ANALYSIS OF A CASE STUDY: THE ATMOSPHERIC COMPONENT}

The case study considered in this paper concerns the monitoring of the deformation induced by dewatering, to allow the construction of a tunnel. The analysed dataset includes 78 descending IWS SLC Sentinel-1A images, which cover the period from March 2015 to November 2017. The analysed dataset includes 1813 interferograms, which were generated using all possible image combinations, with a limit of 1 year for the temporal baselines. The procedure described in the previous section was applied to this dataset.

In this section we analyse the estimation of the atmospheric component. We first analyse the original phases, prior to estimate and remove the atmospheric component, i.e. the original nonfiltered phases. These phases are the output of Step 5 of the above procedure: there is a phase image in correspondence of each acquisition date of the analysed SAR images. In this case study there are 78 10x2 multi-look unwrapped phase images. The first image values are set to zero because they represent the temporal reference.

The objective of the analysis, which was focused on the stable areas, was characterizing the atmospheric phase component of the original data. The analysis was based on this assumption: the phase component related to the residual topographic error, the thermal phase component and the phase noise are spatially uncorrelated. With such an assumption, over the stable areas, there is only one spatially correlated phase component: the atmospheric component.

Therefore, we analysed the spatial correlation of the 78 multilook unwrapped phase images. We used for this the Empirical Autocorrelation Function, EAF, see for details Crosetto et al (2001). An example of EAF is shown in Figure 1. From this function, the following parameter can be derived:

$1 \sigma_{-}$tot is the total standard deviation of the analysed phase image;

$2 \sigma_{-}$corr is the standard deviation of the spatially correlated part of the phase image;

$3 \sigma$ noise is the standard deviation of the spatially uncorrelated part of the phase image;

4 L_corr is the correlation length, i.e. the distance from the origin where the EAF has a correlation which is half of that in the origin.

The main results of the analysis can be summarised as follows. The $31.1 \%$ of the phase images has $\sigma$ corr below $0.4 \mathrm{rad}$. This represents a weak atmospheric phase component. Transformed in displacements, the above standard deviation corresponds to a standard deviation below $1.76 \mathrm{~mm}$. This group is characterised by zero or negligible L_corr. It is interesting to note that the majority of the images of this group (58.3\%) were acquired during winter time or during late autumn. This period is characterized by a reduced atmospheric turbulence. 


\begin{tabular}{|c|c|c|c|c|c|c|}
\hline & \multicolumn{3}{|c|}{ Original non-filtered phases } & \multicolumn{3}{|c|}{ Residual filtered phases } \\
\hline Image \# & $\sigma \_$tot & $\sigma \_$corr & L_corr & $\sigma \_$tot & $\sigma \_$corr & L_corr \\
\hline 3 & 0.71 & 0.60 & 204 & 0.64 & 0.52 & 18 \\
\hline 4 & 0.78 & 0.54 & 0 & 0.67 & 0.44 & 0 \\
\hline 12 & 1.33 & 1.21 & 468 & 1.10 & 0.97 & 384 \\
\hline 14 & 1.01 & 0.92 & 1496 & 0.53 & 0.37 & 0 \\
\hline 16 & 0.72 & 0.56 & 281 & 0.54 & 0.35 & 0 \\
\hline 23 & 0.76 & 0.69 & 1029 & 0.47 & 0.35 & 18 \\
\hline 24 & 0.71 & 0.61 & 1785 & 0.40 & 0.22 & 0 \\
\hline 34 & 0.63 & 0.50 & 204 & 0.60 & 0.47 & 192 \\
\hline 35 & 0.77 & 0.67 & 765 & 0.67 & 0.55 & 329 \\
\hline 36 & 0.83 & 0.72 & 1029 & 0.55 & 0.38 & 0 \\
\hline 37 & 0.74 & 0.59 & 24 & 0.55 & 0.36 & 0 \\
\hline 39 & 0.69 & 0.54 & 26 & 0.63 & 0.47 & 18 \\
\hline 40 & 0.67 & 0.54 & 655 & 0.48 & 0.32 & 0 \\
\hline 41 & 0.94 & 0.80 & 1122 & 0.59 & 0.40 & 0 \\
\hline 42 & 0.80 & 0.63 & 28 & 0.62 & 0.41 & 0 \\
\hline 44 & 1.40 & 1.30 & 638 & 0.98 & 0.85 & 185 \\
\hline 45 & 0.67 & 0.50 & 27 & 0.62 & 0.44 & 18 \\
\hline 48 & 0.69 & 0.55 & 417 & 0.62 & 0.48 & 18 \\
\hline 57 & 0.99 & 0.75 & 26 & 0.81 & 0.53 & 0 \\
\hline 62 & 0.64 & 0.51 & 255 & 0.64 & 0.51 & 183 \\
\hline 64 & 1.16 & 0.95 & 51 & 1.07 & 0.86 & 18 \\
\hline 67 & 0.97 & 0.82 & 842 & 0.64 & 0.45 & 0 \\
\hline 68 & 0.71 & 0.51 & 24 & 0.69 & 0.49 & 0 \\
\hline 69 & 0.69 & 0.56 & 468 & 0.52 & 0.36 & 0 \\
\hline 70 & 1.17 & 1.08 & 1658 & 0.60 & 0.46 & 18 \\
\hline 72 & 0.67 & \begin{tabular}{l|l}
0.54 \\
\end{tabular} & 417 & 0.49 & 0.33 & 0 \\
\hline 76 & 0.76 & 0.63 & 553 & 0.59 & 0.43 & 37 \\
\hline
\end{tabular}

Table 1. Characterization of a set of phase images: original non-filtered phases versus the residual filtered phases

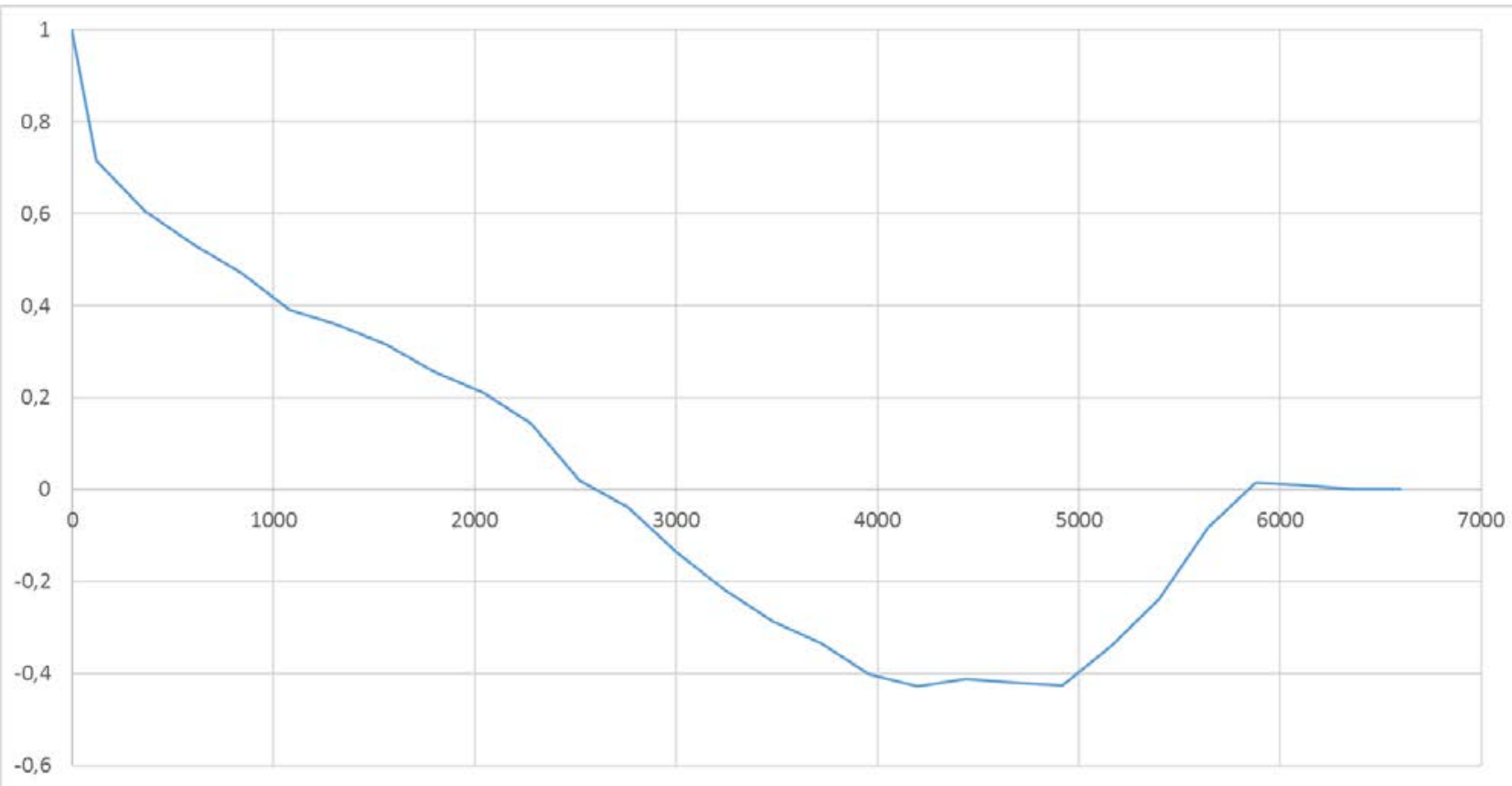

Figure 1. Example of EAF, which corresponds to a phase image (before atmospheric correction) with L_corr equal to $720 \mathrm{~m}$ 
$33.8 \%$ of the images have $\sigma \_$corr that ranges between 0.4 and 0.5 rad. With the exception of one image, this group is characterised by moderated L_corr values, that typically range between 20 and $50 \mathrm{~m}$.

The last group represents the $35 \%$ of the phase images. Its main characteristics are summarized in Table 1 . They have $\sigma_{-}$corr values above $0.5 \mathrm{rad}$. Two thirds of these images were acquired during summer time or late spring: this is the period of the year when there is the maximum atmospheric turbulence. The images are in general characterized by large L_corr (the maximum L_corr is $1785 \mathrm{~m}$ ). The atmospheric component of these images is rather strong. In order to obtaining good deformation monitoring results, it has to be properly estimated and removed.

The second part of the analysis concerns the residual filtered phases. We refer to the results displayed in Table 1 . The proposed procedure achieves a significant reduction of the standard deviation of the spatially correlated part of the phase images: the average reduction of the $\sigma$ _corr is $30.5 \%$. However, the most significant parameter is the correlation length: through the atmospheric component estimation and removal, the L_corr values experience a reduction of $90 \%$. This remarkable drop is key to judge the goodness of the proposed method.

In Table 1 there are five images where, after removing the atmospheric component, the L_corr values remain rather high. This occurs in the images number 12, 34, 35, 44 and 62. In these five images the L_corr of the filtered images remains above 180 $\mathrm{m}$. In two cases (images 12 and 44), the corresponding $\sigma$ _corr values are also high. These two images are characterized by strong atmospheric components (the original image phases have L_corr equal to $468 \mathrm{~m}$ and $638 \mathrm{~m}$, respectively). Such atmospheric component cannot be modelled by the used linear atmospheric model. This is a limitation of the proposed procedure: it is easy-to-implement and quite robust. However, it is not sufficient to cover all possible atmospheric component patterns.

When the model is insufficient to describe a given atmospheric component, there are two options. The first one is to discard the highly non-linear "turbulent" image. This can be an option especially when a large set of images is available. There are however cases where a given image cannot be removed. In this case the deformation time series has to be interpreted with attention: in correspondence to the given image, the time series typically shows a spike due to the strong residual atmospheric component.

The last part of the analysis concerns the deformation area of interest, which is a circle with a 1-km radius. The objective was to assess the effects of the residual atmospheric component in the deformation time series (computed in the Step 13 of the proposed PSI procedure).

For this analysis we selected the stable points contained in the area of interest. These points were selected considering those that have an absolute deformation velocity below $1 \mathrm{~mm} / \mathrm{yr}$. With such a selection, the corresponding time series should basically contain a negligible deformation. They only contain the residual atmospheric component, the noise and the thermal effects (the error in the so-called residual topographic effect, estimated in Step 10, is assumed to be negligible). The analysis was performed considering the original dataset of 78 images, i.e. the images 12 and 44 were included were not discarded.

The average total standard deviation of the time series is 1.90 $\mathrm{mm}$. Only 127 out of 3862 time series (i.e. the $3.3 \%$ of the points) have a total standard deviation above $3 \mathrm{~mm}$ : this represents the $3.3 \%$ of the PSs. These statistics were considered satisfactory in the considered deformation monitoring.

\section{ANALYSIS OF A CASE STUDY: DEFORMATION MONITORING RESULTS}

In this section we briefly discuss the quality of the results of the proposed procedure. For this purpose, we consider two examples of deformation time series generated in the considered case study.

The first example is displayed in Figure 2. In the upper figure, it is displayed the deformation time history of a point. In March 2017 the point suffers a sudden deformation of about $8 \mathrm{~mm}$, which is then followed by an uplift to roughly recover to the original height. In the interpretation of this result it was identified that this behaviour is due to aquifer dewatering, and the subsequent recovery of the water level due to the stop of the pumping activity. The start of the piezometric level descent is indicated by a black arrow: it coincides exactly with the occurrence of the main deformation.

To illustrate the performance of the proposed procedure to estimate the atmospheric component, in Figure 2 (below) the above time series is compared with a solution based on spatiotemporal filters, see Devanthéry et al. (2014). A 96-day moving average was used. In this case, it is evident that this solution is basically missing the main drop, providing a biased temporal low-pass solution. The proposed approach is appropriate to monitor sudden deformation, avoiding the risk of missing part of the information.

A second example of deformation time series is shown in Figure 3 . The deformation is plotted together with the temperatures of the scenes at the time of acquisition of each SAR image. There is a strong correlation between deformation and temperature: this is a displacement caused by thermal expansion. This type of result is possible only if the atmospheric contribution is properly removed: this confirms the goodness of the procedure proposed in this work.

\section{CONCLUSIONS}

A new PSI procedure has been described. Its most original aspect is the estimation of the atmospheric phase component using stable areas located in the vicinity of the monitoring area. This approach avoids the assumption that the low-pass spatial deformation is temporally correlated: sudden deformation can properly be estimated. The performances of the procedure have been described in detail, considering a case study. A full characterization of the atmospheric component has been provided, comparing the original non-filtered phases with the residual filtered ones. The effectiveness of the proposed procedure has been highlighted considering two deformation time series examples. A more detailed description of the above results can be found in Crosetto et al. (2018).

The procedure can work with any type of deformation phenomena, provided that its spatial extension is sufficiently small. The larger is the area of interest, the bigger will be the error in the estimation of the atmospheric component over this area. 

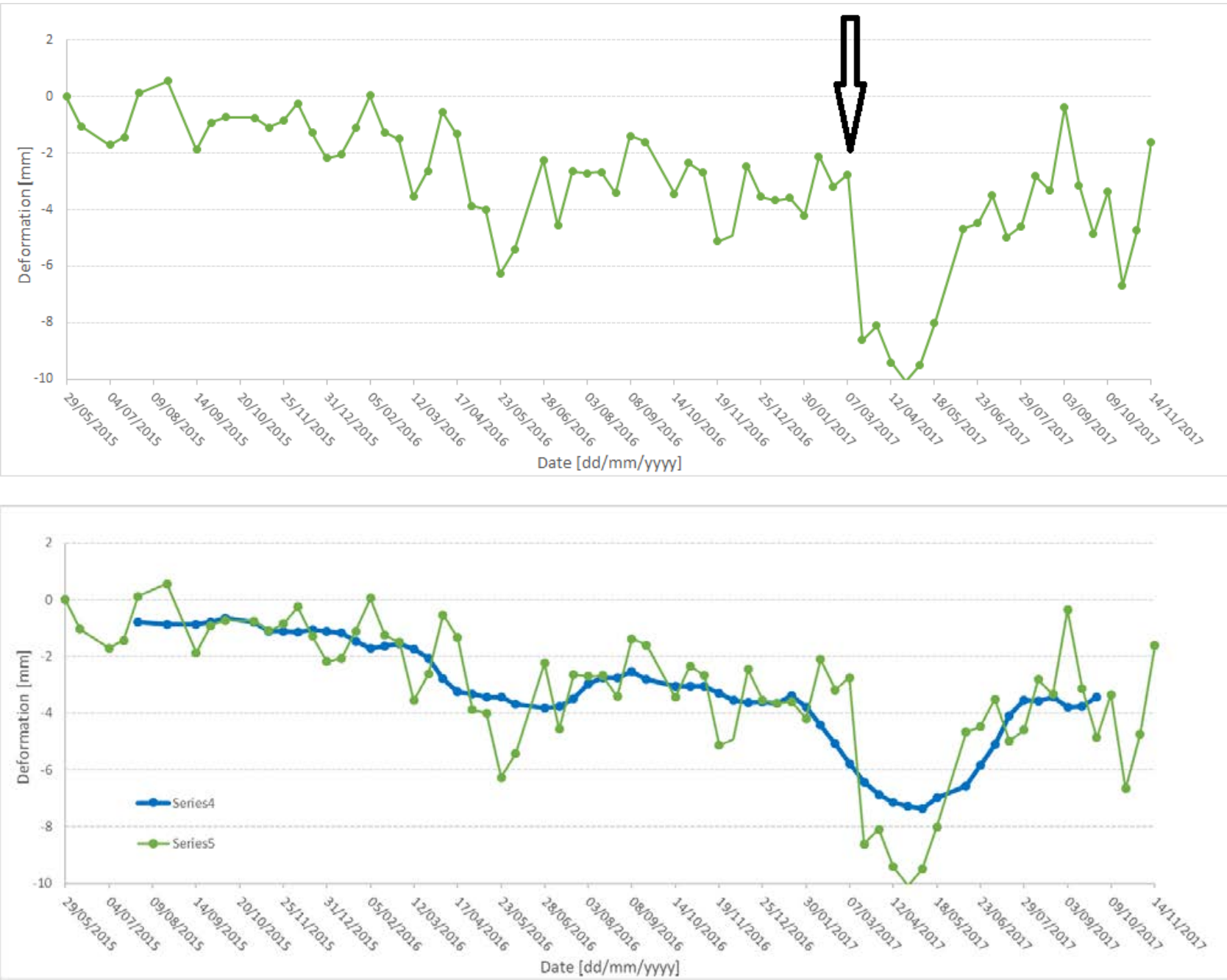

Figure 2. Example of deformation time series that correspond to a sudden deformation, which is then followed by a deformation recovery (above). Low-pass version of the same deformation time series, in blue (below)

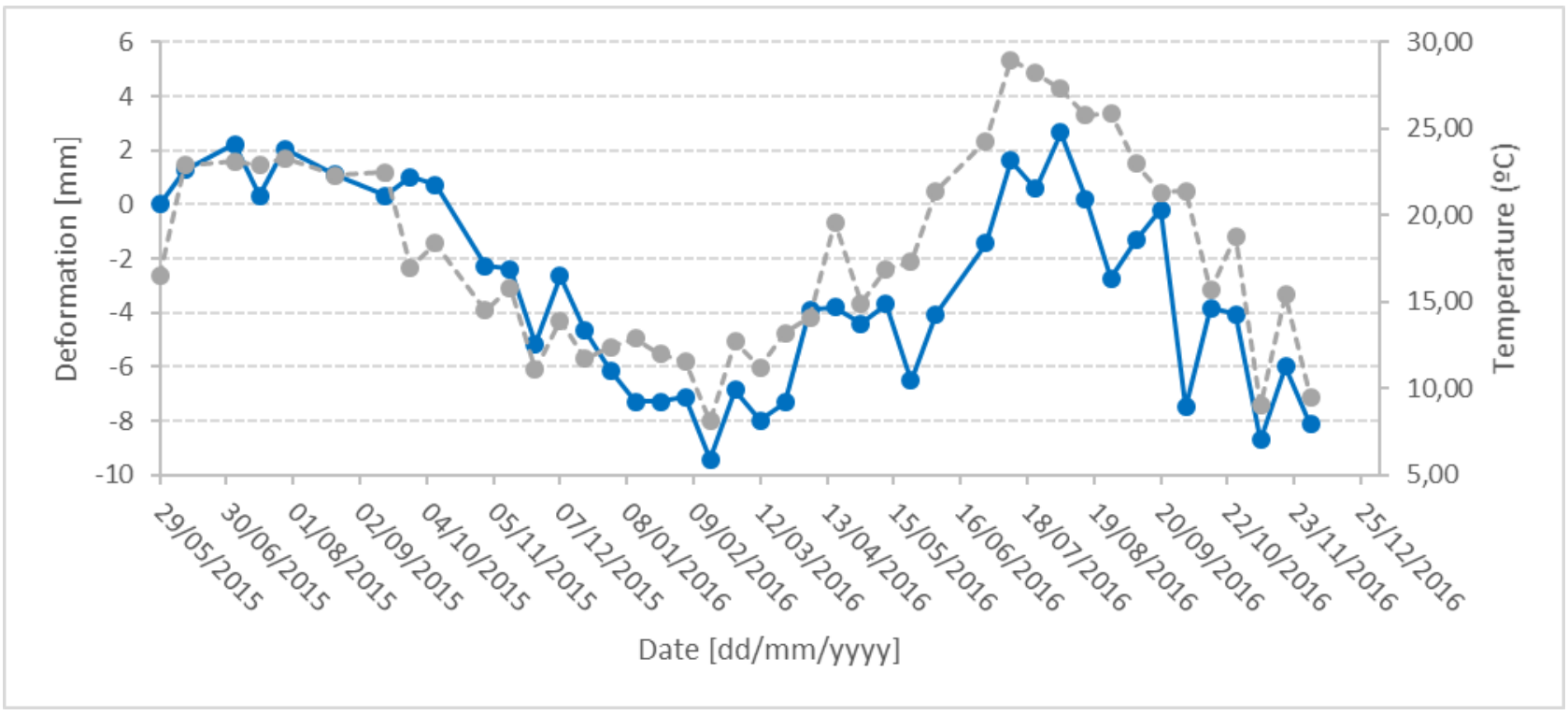

Figure 3. Example of deformation time series that corresponds to a point whose displacements are due to thermal expansion. The deformation (blue line) is plotted together with the temperatures (grey) of the scene in correspondence to the image acquisition dates 


\section{ACKNOWLEDGEMENTS}

This work has been partially funded by the Spanish Ministry of Economy and Competitiveness through the DEMOS project "Deformation monitoring using Sentinel-1 data" (Ref: CGL2017-83704-P).

\section{REFERENCES}

Aslan, G., Cakır, Z., Ergintav, S., Lasserre, C., Renard, F., 2018. Analysis of Secular Ground Motions in Istanbul from a LongTerm InSAR Time-Series (1992-2017). Remote Sens, 10(3), 408.

Bakon, M., Marchamalo, M., Qin, Y., García-Sánchez, A.J., Alvarez, S., Perissin, D., Papco, J., Martínez, R., 2016. Madrid as Seen from Sentinel-1: Preliminary Results. Procedia Comput Sci, 100, 1155-1162.

Berardino, P., Fornaro, G., Lanari, R., Sansosti, E., 2002. A new algorithm for surface deformation monitoring based on small baseline differential SAR interferograms. IEEE Trans Geosci Remote Sens, 40, 2375-2383.

Biescas E., Crosetto, M., Agudo, M., Monserrat, O., Crippa, B., 2007. Two radar interferometric approaches to monitor slow and fast land deformations. Journal of Surveying Engineering, 133 (2), 66-71.

Crosetto, M., Moreno Ruiz, J.A., Crippa, B., 2001. Uncertainty propagation in models driven by remotely sensed data". Remote Sens Environ, 76 (3), 373-385.

Crosetto, M., Monserrat, O., Cuevas, M. Crippa, B., 2011. Spaceborne Differential SAR Interferometry: Data Analysis Tools for Deformation Measurement. Remote Sens, 3, 305-318.

Crosetto, M., Monserrat, O., Cuevas-González, M., Devanthéry, N., Crippa, B., 2016. Persistent Scatterer Interferometry: a review. ISPRS J Photogramm Remote Sens, 115, 78-89.

Crosetto, M., Devanthéry, N., Monserrat, O., Barra, A., CuevasGonzález, M., Mróz, M., Botey-Bassols, J., Vázquez-Suñé, E., Crippa, B., 2018. A Persistent Scatterer Interferometry Procedure Based on Stable Areas to Filter the Atmospheric Component. Remote Sens, 10(11), pp. 1780.

Devanthéry, N., Crosetto, M., Monserrat, O., Cuevas-González, M., Crippa, B., 2014. An approach to Persistent Scatterer Interferometry. Remote Sens, 6, 6662-6679.

Du, Z., Ge, L., Ng, A.H.M., Xiaojing, L., Li, L., 2018. Mapping land subsidence over the eastern Beijing city using satellite radar interferometry. Int J Digital Earth, 11(5), 504-519.

Ferretti, A., Prati, C., Rocca, F., 2000. Nonlinear subsidence rate estimation using permanent scatterers in differential SAR interferometry. IEEE Trans Geosci Remote Sens, 38, 2202-2212.

Ferretti, A., Prati, C., Rocca, F., 2001. Permanent scatterers in SAR interferometry. IEEE Trans Geosci Remote Sens, 39, 8-20.

Fiaschi, S., Tessitore, S., Bonì, R., Di Martire, D., Achilli, V., Borgstrom, S., Ibrahim, A., Floris, M., Meisina, C., Ramondini, M., Calcaterra, D., 2017. From ERS-1/2 to Sentinel-1: two decades of subsidence monitored through A-DInSAR techniques in the Ravenna area (Italy). GIsci Remote Sens, 54(3), 305-328.
Monserrat, O., Crosetto, M., Cuevas, M., Crippa, B., 2011. The Thermal Expansion Component of Persistent Scatterer Interferometry Observations. IEEE Geosci Remote S, 8 (5), 864868 .

Shirzaei, M., Bürgmann, R., Fielding, E.J., 2017. Applicability of Sentinel-1 Terrain Observation by Progressive Scans multitemporal interferometry for monitoring slow ground motions in the San Francisco Bay Area. Geophys Res Lett, 44(6), 2733-2742.

Sowter, A., Amat, M.B.C., Cigna, F., Marsh, S., Athab, A., Alshammari, L., 2016. Mexico City land subsidence in 20142015 with Sentinel-1 IW TOPS: Results using the Intermittent SBAS (ISBAS) technique. Int J Appl Earth Obs Geoinf, 52, 230242.

Yu, L., Yang, T., Zhao, Q., Liu, M., Pepe, A., 2017. The 20152016 Ground Displacements of the Shanghai coastal area Inferred from a combined COSMO-SkyMed/Sentinel-1 DInSAR Analysis. Remote Sens, 9(11), 1194. 